João Paulo Elsen SAUT ${ }^{1}$ Eduardo Harry BIRGEL JUNIOR ${ }^{1}$

\section{Correspondência para:} EDUARDOHARRY BIRGELJUNIOR Departamento de Clínica Médica Faculdade de Medicina Veterinária e Zootecnia

Universidade de São Paulo Av. Prof. Dr. Orlando Marques de Paiva, 87 05508-900 - São Paulo - SP ehbirgel@usp.br

Recebido para publicação: 20/10/2004 Aprovado para publicação: 23/08/2005

\title{
Influência do período pós-parto sobre o leucograma de fêmeas bovinas da raça holandesa
}

1 - Departamento de Clínica Médica da Faculdade de Medicina Veterinária e Zootecnia da Universidade de São Paulo, São Paulo - SP

\section{Resumo}

Para avaliar a influência do período pós-parto sobre os constituintes do leucograma de fêmeas bovinas da raça Holandesa, foram colhidas 142 amostras de sangue nos primeiros 90 dias pós-parto. As amostras foram colhidas de vacas clinicamente sadias e não reagentes ao antígeno (GP 51) do vírus da Leucose dos Bovinos, divididas em 9 grupos experimentais, de acordo com o momento da colheita. Nas amostras de sangue foi realizada a contagem do número total de leucócitos e contagem diferencial de leucócitos. Durante todo o período estudado o quadro leucocitário foi predominantemente linfocitário, sendo nas primeiras 24 horas após aparição observado uma leucocitose fisiológica por neutrofilia com desvio à esquerda regenerativo, linfocitose e eosinopenia. Na primeira semana após o parto ocorreu a diminuição gradual do número de leucócitos, devido à redução do número absoluto de neutrófilos e linfócitos, sendo que exceção feita ao número absoluto de eosinófilos, nas amostras colhidas entre $6-\mid 8$ dias após o parto os valores já estavam nos patamares observados nos animais fora do puerpério. Os valores de referência na primeira semana de puerpério variaram: entre $18.977 \pm 5.644$ e $12.247 \pm 5.058$ leucócitos/ $\mathrm{mm}^{3} ; 8.453 \pm 2.956$ e $4.376 \pm 4.139$ neutrófilos $/ \mathrm{mm}^{3}(532 \pm 547$ e 88 \pm 174 neutófilos bastonete $/ \mathrm{mm}^{3} ; 7.920 \pm 2.689$ e $4.289 \pm 4.039$ neutrófilos segmentado/ $\left.\mathrm{mm}^{3}\right) ; 334 \pm 387$ e $225 \pm 298$ eosinófilos $/ \mathrm{mm}^{3} ; 50 \pm 128$ e 23 \pm 45 basófilos $/ \mathrm{mm}^{3} ; 10.004 \pm 4.901$ e $7.595 \pm 3.127$ linfócitos $/ \mathrm{mm}^{3} ; 121$ \pm 178 e $21 \pm 85$ monócitos $/ \mathrm{mm}^{3}$.

\section{Introdução}

Na literatura sobre hematologia existe a opinião concordante, quase unânime, que animais criados sob diferentes condições ambientais, climáticas e de manejo apresentariam evidentes variações dos elementos constituintes sangüíneos ${ }^{1}$. Da mesma forma, as variações observadas no hemograma de bovinos acometidos por anaplasmose e babesiose são consideradas de fundamental importância, particularmente, para a interpretação do leucograma de bovinos criados em regiões tropicais e subtropicais, como o Brasil, pois nestas regiões os bovinos são naturalmente premunidos contra o Anaplasma sp e a Babesia spp, condição que determina uma leucocitose por linfocitose ${ }^{1}$. Assim sendo, os valores obtidos para os animais criados em uma região não podem ser considerados, sem uma adequada avaliação, como padrão de referência fora dessa região, devendo ser estabelecidos padrões de referência brasileiros para o hemograma dos bovinos aqui criados ${ }^{1}$.

Diante dessa necessidade, há algumas décadas atrás, uma série de pesquisas para estabelecer o quadro hemático dos bovinos e que atendessem ou satisfizessem as necessidades nacionais foram iniciadas por pesquisadores da Clínica de Bovinos do Centro de Pesquisa e Diagnóstico de Enfermidades de Ruminantes do Departamento de Clínica Médica da Faculdade de Medicina Veterinária e 
Zootecnia da Universidade de São Paulo. Dentre essas pesquisas devem ser destacadas aquelas dissertações e teses preocupadas com o estabelecimento dos valores de referência do leucograma de bovinos das raças Holandesa ${ }^{2}$, Jersey ${ }^{1}$, Nelore ${ }^{3}$, Gir $^{2}$ e Girolando ${ }^{2}$.

Ao compulsar a literatura observouse que estudos relacionados à influência do puerpério sobre o leucograma de bovinos não mereceram, ainda, maior atenção de pesquisadores, pois foram encontradas apenas onze pesquisas. Ao analisar essas pesquisas verificou-se que somente nas pesquisas de Straub et al. ${ }^{8}$, Guidry, Paape e Pearson $^{6}$, D'angelino et al. ${ }^{4}$, Hussain e Daniel ${ }^{7}$ e Fagliari et $\mathrm{al}^{5}$ todos os constituintes do leucograma foram determinados, sendo os seus valores apresentados em números absolutos.

A análise da literatura, que procurou avaliar a influência do período pós-parto no leucograma de bovinos, evidenciou que houve pequena preocupação relacionada ao mencionado tema, pois foram encontradas somente treze pesquisas sobre $\mathrm{O}$ assunto $^{4,5,6,7,8,9,10,11,12,13,14,15,16}$. Ao analisar essas pesquisas verificou-se que somente cinco delas ${ }^{4,5,6,7,8}$ todos os constituintes do leucograma foram determinados, sendo os seus valores apresentados em números absolutos. Verificou-se, ainda, que o quadro leucocitário foi acompanhado, detalhadamente, durante toda a evolução do puerpério, em apenas uma pesquisa ${ }^{14}$, sendo que em algumas delas esse acompanhamento ocorreu somente nos primeiros 7 dias do puerpério ${ }^{8,13}$, semanalmente ${ }^{10,12,16}$, quinzenalmente ${ }^{5}$ ou ainda de forma mais esporádica 4,6,7,9,11,15.

Destas pesquisas somente duas foram conduzidas utilizando-se animais criados nos Brasil ${ }^{4,5}$, porém o leucograma não foi avaliado com uma periodicidade que permitisse avaliar detalhadamente a influência do período pós-parto no quadro leucocitário ou a necessidade de utilizar-se valores de referência específicos nesse período para que tal exame laboratorial possa ser utilizado na sua plenitude no auxílio do estabelecimento de diagnóstico das enfermidades do puerpério.

Os fatos anteriormente apontados evidenciam, de forma notória, a necessidade da realização da presente pesquisa que tem por objetivo determinar os valores de referência do leucograma de fêmeas bovinas criadas nas condições brasileiras de manejo e alimentação, bem como a avaliação da influência do período pós-parto sobre o leucograma nos primeiros 90 dias pós-parto.

\section{Materiais e Métodos}

No período compreendido entre a parição e os 90 dias após o parto foram colhidas 142 amostras de sangue de fêmeas

Tabela 1 - Constituição dos grupos experimentais para avaliação da influência do período pós-parto sobre os constituintes do leucograma de vacas da raça Holandesa - São Paulo - 2004

\begin{tabular}{ccll}
\hline Grupo & N & \multicolumn{2}{c}{ Momento de colheita das amostras } \\
\hline I & 15 & $0-\mid 1^{\circ}$ dia pós-parto & $(0-\mid 24$ horas pós-parto $)$ \\
II & 9 & $1^{\circ}-\mid 2^{\circ}$ dia pós-parto & $(24-\mid 48$ horas pós-parto $)$ \\
III & 8 & $2^{\circ}-\mid 4^{\circ}$ dia pós-parto & $(48-\mid 96$ horas pós-parto $)$ \\
IV & 14 & $4^{\circ}-\mid 6^{\circ}$ dia pós-parto & $(96-\mid 144$ horas pós-parto $)$ \\
V & 17 & $6^{\circ}-\mid 8^{\circ}$ dia pós-parto & $(144-\mid 192$ horas pós-parto $)$ \\
VI & 16 & $8^{\circ}-\mid 15^{\circ}$ dia pós-parto & $(192-\mid 360$ horas pós-parto $)$ \\
VII & 14 & $15^{\circ}-\mid 30^{\circ}$ dia pós-parto & $(360-\mid 720$ horas pós-parto $)$ \\
VIII & 29 & $30^{\circ}-\mid 60^{\circ}$ dia pós-parto & $(720-\mid 1440$ horas pós-parto $)$ \\
IX & 20 & $60^{\circ}-\mid 90^{\circ}$ dia pós-parto & $(1440-\mid 2160$ horas pós-parto $)$ \\
\hline
\end{tabular}

$\mathrm{N}=$ número de animais 
bovinas da raça Holandesa provenientes de 3 propriedades leiteiras do tipo A e 8 do tipo $\mathrm{B}$, livres de brucelose e tuberculose e não reagentes aos antígenos do vírus da Leucose dos Bovinos. De acordo com o momento da colheita, essas amostras foram agrupadas em 9 momentos conforme a seguir discriminado na tabela 1 .

As amostras de sangue foram colhidas por punção da veia jugular externa, utilizando-se um sistema para colheita a vácuo constituído de agulhas $25 \times 8 \mathrm{~mm}$ (21G) para múltipla colheita, acopladas a tubos siliconizados, contendo uma solução aquosa de etileno diamino tetracetato tripotássico

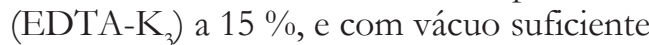
para aspirar 4,5 $\mathrm{ml}$ de sangue, sendo realizadas as seguintes provas: contagem do número total de leucócitos e contagem diferencial de leucócitos.

A contagem do número total de leucócitos foi realizada em Câmara de Neubauer modificada, sendo as amostras de sangue diluídas, em pipeta hematimétrica específica, na proporção de 1:20, utilizandose como solução diluidora o líquido de Thoma de acordo com as recomendações de Birge ${ }^{17}$. Com o sangue "in natura" foram distendidos dois esfregaços sangüíneos destinados à contagem diferencial de leucócitos. Esses esfregaços, após secarem, foram corados utilizando-se o corante de Rosenfeld, segundo técnica padronizada para os animais por Birgel ${ }^{17}$. Em cada esfregaço sangüíneo foram diferenciados 100 leucócitos e classificados, de acordo com suas características morfológicas e tintoriais, em neutrófilos com núcleo em bastonete, neutrófilos com núcleo segmentado, eosinófilos, basófilos, linfócitos e monócitos.

A pesquisa de anticorpos séricos antivírus da Leucose dos Bovinos, para detecção dos animais não reagentes, foi realizada através da prova de imunodifusão radial dupla de Ouchterlony em gel de ágar, utilizando-se antígeno glicoprotéico (gp 51) da cápsula do Vírus da Leucose dos Bovinos, segundo metodologia padronizada por Birgel $^{18}$ e modificada por D'Angelino ${ }^{19}$.
Para calcular os valores da média aritmética e o desvio padrão, assim como avaliar a influência do puerpério sobre os elementos constituintes do leucograma, utilizou-se o programa de computador $\mathrm{SAS}^{20}$, aplicando-se, inicialmente, análise de variância, sendo, a seguir, utilizado para comparação entre os pares de médias o teste de Tukey's, com nível de significância igual a $5 \%$, conforme recomendaram Berquó, Souza e Gotlieb ${ }^{21}$.

\section{Resultados}

Na presente pesquisa foi demonstrada a influência dos fatores relacionadas à parição sobre o leucograma de bovinos da raça Holandesa, sendo que os resultados obtidos estão detalhados nas tabelas 2 e 3 e Figura 1. No delineamento experimental proposto, verificou-se que as variações observadas no leucograma de bovinos da raça Holandesa foram decorrentes, particularmente, às alterações no número de neutrófilos, linfócitos e eosinófilos.

Durante a evolução do puerpério fisiológico, o número total de leucócitos observado em amostras de sangue colhidas nas primeiras 24 horas após o parto (18.977 \pm 5.644 leucócitos $/ \mathrm{mm}^{3}$ ) diminuíram, gradativamente, atingindo valores iguais a $12.247 \pm 5.058$ leucócitos $/ \mathrm{mm}^{3} \mathrm{em}$ amostras colhidas entre $6-\mid 8$ dias após o parto. A partir desse momento, o número total de leucócitos oscilaram entre $10.764 \pm$ 2.848 leucócitos/ $\mathrm{mm}^{3}$ e $12.106 \pm 4.892$ células $/ \mathrm{mm}^{3}$ sem que qualquer tendência de aumento ou diminuição pudesse ser verificada.

A análise dos resultados apresentados na tabela 2 e figura 1 demonstrou que o número absoluto de neutrófilos sofreu influência do puerpério, sendo que os valores obtidos em amostras colhidas com menos de 24 horas após o parto $\left(8.453 \pm 2.956\right.$ neutrófilos $\left./ \mathrm{mm}^{3}\right)$ diminuíram gradativamente nos primeiros dias do puerpério, atingindo valores iguais a 4.602 \pm 2.761 neutrófilos $/ \mathrm{mm}^{3}$ em amostras colhidas entre $2-\mid 4$ dias após o parto. A partir desse momento o número total de 
neutrófilos deixou de sofrer influência, passando a oscilarem entre $3.171 \pm 1.390$ neutrófilos $/ \mathrm{mm}^{3}$ e $5.105 \pm 2.747$ neutrófilos $/ \mathrm{mm}^{3}$.

Os resultados da presente pesquisa demonstraram que os neutrófilos com núcleo segmentado representam a maioria dessas células, variando a sua freqüência entre $97,7 \%$ e $100 \%$ dos tipos de neutrófilos encontrados nos diversos grupos experimentais, sendo que os neutrófilos com núcleo em forma de bastonete representam entre 0 e $6,7 \%$ de todos os neutrófilos observados no período estudado. Durante todo o período pesquisado não foram encontradas na circulação, em nenhuma ocasião, células imaturas dessa série de granulócitos. As variações observadas durante o puerpério recente para o número absoluto de neutrófilos do tipo bastonete e de neutrófilos com núcleo segmentado eram semelhantes àquelas descritas para o número total de neutrófilos, isto é, durante o evoluir do período pós-parto ocorre a gradual diminuição dos seus valores.

Conforme apresentado na tabela 3, o número absoluto de neutrófilos com núcleo em forma de bastonete, também, sofreu variações semelhantes àquelas relatadas para o número total de leucócitos e de neutrófilos, isto é, ocorreu uma gradual diminuição dos seus valores nos primeiros dias do puerpério. Os valores absolutos de neutrófilos com núcleo em forma de bastonete, encontrados nas amostras colhidas com menos de 24 horas após o parto, iguais a $532 \pm 547$ neutrófilos bastonetes $/ \mathrm{mm}^{3}$ diminuíram com a evolução do período pósparto, A partir das amostras colhidas entre 4 - 6 dias após o parto, o número absoluto de neutrófilos do tipo bastonete deixou de sofrer influência do puerpério, sendo que os valores passaram a oscilar entre $0 \pm 0$ neutrófilos bastonete $/ \mathrm{mm}^{3}$ e $88 \pm 174$ neutrófilos bastonete $/ \mathrm{mm}^{3}$.

O número absoluto de neutrófilos com núcleo segmentado encontrados nas amostras colhidas com menos de 24 horas após o parto, iguais a $7.920 \pm 2.689$ neutrófilos segmentado $/ \mathrm{mm}^{3}$ diminuíram com a evolução do puerpério, deixando de sofrer influência nos seus valores nas amostras

Tabela 2 - Avaliação da influência do período pós-parto no leucograma de bovinos da raça Holandesa. São Paulo - 2004

\begin{tabular}{|c|c|c|c|c|c|c|}
\hline \multicolumn{7}{|c|}{ Leucograma } \\
\hline $\begin{array}{l}\text { Período } \\
\text { Pós-parto } \\
\text { (dias } \\
\text { pós- } \\
\text { parto) }\end{array}$ & $\begin{array}{l}\text { Leucócitos } \\
\text { (células/ } \\
\mathrm{mm}^{3} \text { ) }\end{array}$ & $\begin{array}{c}\text { Neutrófilos } \\
\text { (células/ } \\
\mathrm{mm}^{3} \text { ) }\end{array}$ & $\begin{array}{c}\text { Eosinófilos } \\
\text { (células/ } \\
\mathrm{mm}^{3} \text { ) }\end{array}$ & $\begin{array}{c}\text { Basófilos } \\
\text { (células/ } \\
\mathrm{mm}^{3} \text { ) }\end{array}$ & $\begin{array}{c}\text { Linfócitos } \\
\text { (células/ } \\
\mathrm{mm}^{3} \text { ) }\end{array}$ & $\begin{array}{c}\text { Monócitos } \\
\text { (células/m } \\
\mathrm{m}^{3} \text { ) }\end{array}$ \\
\hline $0-\mid 1$ dia & $\begin{array}{r}18.977 \\
5.644^{\mathrm{a}}\end{array}$ & $\begin{array}{c}8.453 \pm \\
2.956^{\mathrm{a}}\end{array}$ & $334 \underset{\text { abd }}{ \pm} 387$ & $50 \pm 128^{a b}$ & $\begin{array}{r}10.004 \pm \\
4.901^{\mathrm{a}}\end{array}$ & $121_{\mathrm{ab}}^{ \pm} 178$ \\
\hline $\begin{array}{c}1-\mid 2 \\
\text { dias }\end{array}$ & $\begin{array}{c}13.150 \pm \\
1.647^{\mathrm{b}}\end{array}$ & $\begin{array}{c}5.399 \pm \\
2.387^{\mathrm{b}}\end{array}$ & $138 \pm 159^{\mathrm{a}}$ & $46 \pm 138^{a b}$ & $\begin{array}{l}7.366 \pm \\
2.481^{\mathrm{ab}}\end{array}$ & $201 \pm 236$ \\
\hline $\begin{array}{c}2-\mid 4 \\
\text { dias }\end{array}$ & $\begin{array}{c}15.875 \pm \\
6.566^{\mathrm{abc}}\end{array}$ & $\begin{array}{l}4.602 \pm \\
2.761^{\mathrm{bc}}\end{array}$ & $506 \underset{\text { bcd }}{ \pm} 315$ & $64 \pm 123^{a b}$ & $\begin{array}{c}10.395 \pm \\
4.139^{\mathrm{a}}\end{array}$ & $122 \pm 136$ \\
\hline $\begin{array}{c}4-\mid 6 \\
\text { dias }\end{array}$ & $\begin{array}{c}14.761 \\
5.362^{\mathrm{b}}\end{array}$ & $\begin{array}{l}5.105 \pm \\
2.747^{\mathrm{bd}}\end{array}$ & $128 \pm 152^{\mathrm{a}}$ & $99 \pm 184^{\mathrm{ab}}$ & $\begin{array}{c}9.262 \pm \\
3.644^{\mathrm{a}}\end{array}$ & $159 \pm 225^{\mathrm{a}}$ \\
\hline $\begin{array}{c}6-\mid 8 \\
\text { dias }\end{array}$ & $\begin{array}{c}12.247 \pm \\
5.058^{\mathrm{bc}}\end{array}$ & $\begin{array}{l}4.376 \pm \\
4.139^{\mathrm{bc}}\end{array}$ & $225 \underset{\mathrm{ab}}{ \pm} 298$ & $23 \pm 45^{b}$ & $\begin{array}{l}7.595 \pm \\
3.127^{\mathrm{ab}}\end{array}$ & $21 \pm 85^{b}$ \\
\hline $\begin{array}{c}8-\mid 15 \\
\text { dias }\end{array}$ & $\begin{array}{c}12.106 \pm \\
4.892^{\mathrm{bc}}\end{array}$ & $\begin{array}{l}3.825 \pm \\
2.163^{\mathrm{bc}}\end{array}$ & $325 \underset{\text { abd }}{ \pm} 412$ & $53 \pm 78^{a b}$ & $\begin{array}{l}7.865 \pm \\
4.114^{\mathrm{ab}}\end{array}$ & $58 \pm 101^{a b}$ \\
\hline $\begin{array}{c}15-\mid 30 \\
\text { dias }\end{array}$ & $\begin{array}{c}11.211 \pm \\
3.813^{\mathrm{bc}}\end{array}$ & $\begin{array}{l}3.313 \pm \\
1.797^{\mathrm{cd}}\end{array}$ & $516 \underset{\text { abcd }}{ \pm} 682$ & $19 \pm 42^{b}$ & $\begin{array}{l}7.354 \pm \\
2.950 \mathrm{ab}\end{array}$ & $51 \pm 80^{a b}$ \\
\hline $\begin{array}{c}30-\mid 60 \\
\text { dias }\end{array}$ & $\begin{array}{c}10.764 \pm \\
2.848^{c}\end{array}$ & $\begin{array}{l}3.574 \pm \\
1.290^{\mathrm{bd}}\end{array}$ & $419 \pm 295$ & $80 \pm 114^{a}$ & $\begin{array}{l}6.659 \pm \\
2.206^{\mathrm{b}}\end{array}$ & $27 \pm 70^{b}$ \\
\hline $\begin{array}{c}60-\mid 90 \\
\text { dias }\end{array}$ & $\begin{array}{c}10.973{ }^{ \pm} \\
2.034^{\mathrm{c}}\end{array}$ & $\begin{array}{l}3.171 \pm \\
1.390^{\mathrm{cd}}\end{array}$ & $801 \pm 717^{c}$ & $76 \pm 90^{a}$ & $\begin{array}{c}6.821 \pm \\
2.027^{b}\end{array}$ & $104 \pm 120^{a}$ \\
\hline
\end{tabular}


Tabela 3 - Avaliação da influência do período pós-parto no número absoluto de neutrófilos de bovinos da raça Holandesa. São Paulo - 2004

\begin{tabular}{cccc}
\hline \multirow{2}{*}{$\begin{array}{c}\text { Período pós-parto } \\
\text { (dias pós-parto) }\end{array}$} & $\begin{array}{c}\text { Bastonete } \\
\text { (células/ } \mathrm{mm}^{3} \text { ) }\end{array}$ & $\begin{array}{c}\text { Segmentado } \\
\text { (células/ } \mathrm{mm}^{3} \text { ) }\end{array}$ & $\begin{array}{c}\text { Total } \\
\text { (células/ } \mathrm{mm}^{3} \text { ) }\end{array}$ \\
\hline $0-\mid 1$ dia & $532 \pm 547^{\mathrm{b}}$ & $7.920 \pm 2.689^{\mathrm{a}}$ & $8.453 \pm 2.956^{\mathrm{a}}$ \\
$1-\mid 2$ dias & $200 \pm 195^{\mathrm{b}}$ & $5.199 \pm 2.369^{\mathrm{b}}$ & $5.399 \pm 2.387^{\mathrm{b}}$ \\
$2-\mid 4$ dias & $98 \pm 124^{\mathrm{bc}}$ & $4.504 \pm 2.822^{\mathrm{bc}}$ & $4.602 \pm 2.761^{\mathrm{bc}}$ \\
$4-\mid 6$ dias & $157 \pm 193^{\mathrm{b}}$ & $4.948 \pm 2.729^{\mathrm{bd}}$ & $5.105 \pm 2.747^{\mathrm{bd}}$ \\
$6-\mid 8$ dias & $88 \pm 174^{\mathrm{bc}}$ & $4.289 \pm 4.039^{\mathrm{bc}}$ & $4.376 \pm 4.139^{\mathrm{bc}}$ \\
$8-\mid 15$ dias & $10 \pm 28^{\mathrm{c}}$ & $3.815 \pm 2.172^{\mathrm{bc}}$ & $3.825 \pm 2.163^{\mathrm{bc}}$ \\
$15-\mid 30$ dias & $18 \pm 48^{\mathrm{c}}$ & $3.925 \pm 1.774^{\mathrm{bc}}$ & $3.313 \pm 1.797^{\mathrm{cd}}$ \\
$30-\mid 60$ dias & $2 \pm 14^{\mathrm{c}}$ & $3.572 \pm 1.294^{\mathrm{cd}}$ & $3.574 \pm 1.290^{\mathrm{bd}}$ \\
$60-\mid 90$ dias & $0 \pm 0^{\mathrm{c}}$ & $3.171 \pm 1.390^{\mathrm{c}}$ & $3.171 \pm 1.390^{\mathrm{cd}}$ \\
\hline
\end{tabular}

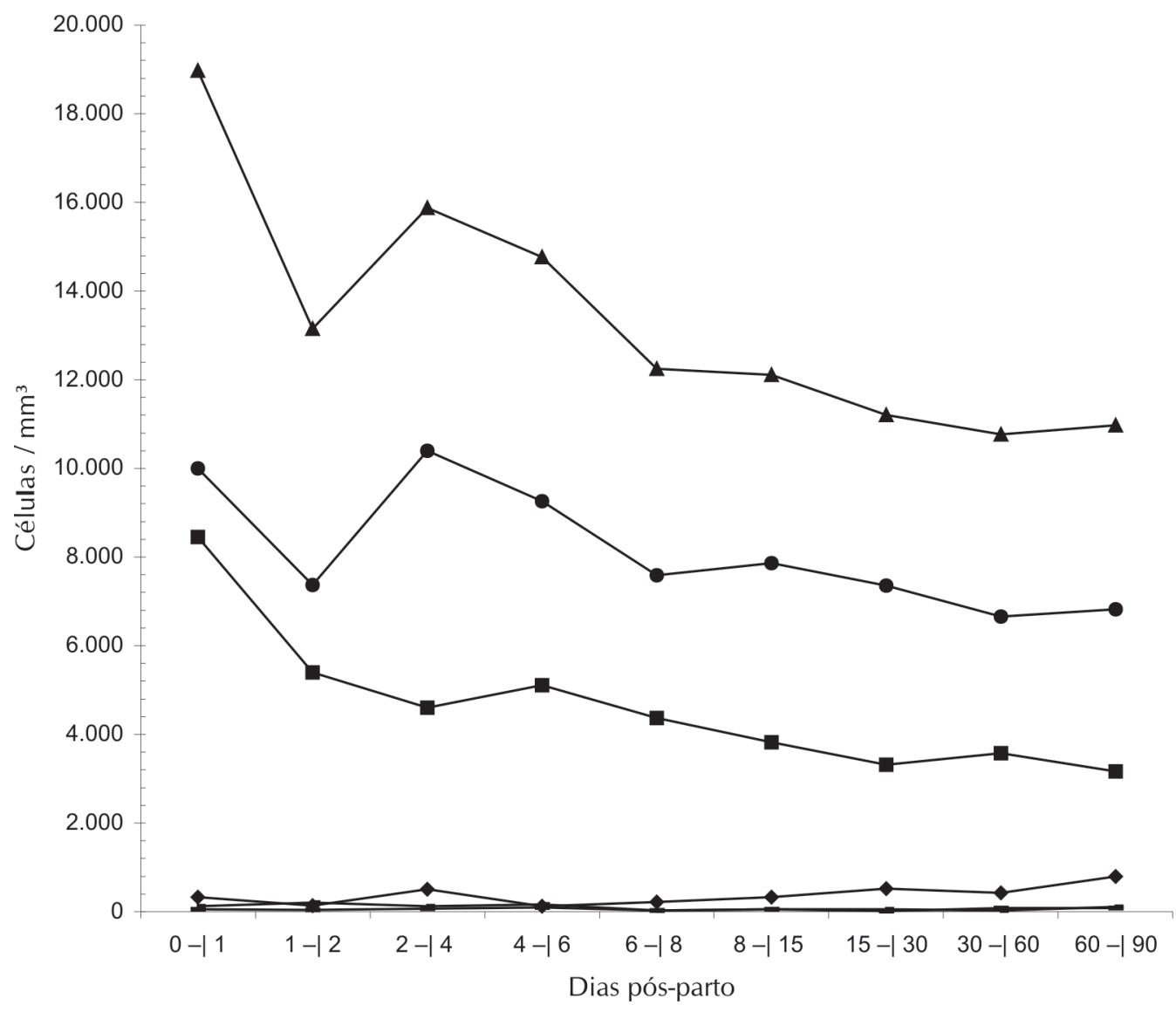

Figura 1 - Influência do período pós-parto no leucograma de bovinos da raça Holandesa. São Paulo - 2004 
colhidas entre $4-\mid 6$ dias após o parto, a partir desse momento os valores do número absoluto de neutrófilos segmentado passaram a oscilar entre $3.171 \pm 1.390$ neutrófilos segmentados $/ \mathrm{mm}^{3}$ e $4.289 \pm$ 4.039 neutrófilos segmentados $/ \mathrm{mm}^{3}$.

A avaliação dos resultados apresentados na tabela 2 e figura 1 para o número de linfócitos, demonstrou que o quadro leucocitário foi predominantemente linfocitário e que houve significativa influência do pós-parto sobre essa variável. Os valores obtidos em amostras colhidas com menos de 24 horas após o parto $(10.004 \pm 4.901$ linfócitos $/ \mathrm{mm}^{3}$ ) diminuíram gradativamente nos primeiros dias do puerpério atingindo valores iguais a $7.595 \pm$ 3.127 linfócitos $/ \mathrm{mm}^{3}$ nas amostras colhidas entre $6-\mid 8$ dias após o parto. A partir desse momento, o número absoluto de linfócitos deixou de sofrer influência na evolução do período pós-parto, passando a oscilarem entre $6.659 \pm 2.206$ linfócitos $/ \mathrm{mm}^{3}$ e 7.865 \pm 4114 linfócitos $/ \mathrm{mm}^{3}$, sem que qualquer tendência de aumento ou diminuição pudesse ser verificada.

Os resultados apresentados na tabela 2 e fig 1 para o número absoluto de eosinófilos demonstraram haver influência do período pós-parto sobre essa variável. Após uma fase inicial, na qual os valores oscilaram entre $138 \pm 159$ eosinófilos $/ \mathrm{mm}^{3}$ e $506 \pm 315$ eosinófilos $/ \mathrm{mm}^{3}$, observouse a partir do quarto dia após o parto um gradual aumento dos valores obtidos para esta variável, passando o número absoluto de eosinófilos de $128 \pm 152$ eosinófilos / $\mathrm{mm}^{3}$, em amostras colhidas entre $4-\mid 6$ dias após o parto, para $801 \pm 717$ eosinófilos $/ \mathrm{mm}^{3} \mathrm{em}$ amostras colhidas entre $60-\mid 90$ dias após o parto.

Analisando os resultados para o número absoluto de basófilos, verificou-se na comparação de diversos pares de médias, a existência de diferenças estatisticamente significantes. Entretanto, não foi possível a determinação da natureza dessa influência, pois os valores do número absoluto de basófilos oscilaram entre $19 \pm 42$ células / $\mathrm{mm}^{3}$ e $99 \pm 184$ células $/ \mathrm{mm}^{3}$, sem que qualquer tendência de aumento ou diminuição dos valores pudesse ser verificada durante a evolução do período pós-parto acompanhado.

De forma semelhante, ao se analisar os resultados encontrados para o número absoluto de monócitos, foram encontrados na comparação de diversos pares de média, a existência de diferenças estatísticas significativas sem que a natureza dessa influência pudesse ser determinada, pois durante a evolução do período pós-parto os valores do número absoluto de monócitos oscilaram entre $21 \pm 85$ monócitos $/ \mathrm{mm}^{3}$ e $201 \pm 236$ monócitos / $\mathrm{mm}^{3}$, sem que qualquer tendência de aumento ou diminuição dos valores pudesse ser verificada.

\section{Discussão}

Ao se confrontar os resultados descritos na pesquisa com os encontrados na literatura compulsada, constatou-se a existência de quase absoluta concordância entre os autores de que a parição determinava intensas modificações no leucograma $1,5,7,8,13,14,16,22,23$, sendo que essas alterações, caracterizadas por leucocitose devido a neutrofilia e eosinopenia, já podiam ser observadas nos últimos dias de gestação $0^{6,13,22,23}$ e que estavam relacionadas ao aumento da secreção de hormônios da córtex adrenal, observado no final da gestação, durante a parição e / ou indução fisiológica do parto ${ }^{6,8,13,24}$.

Dessa forma, os resultados obtidos nesta pesquisa, nas primeiras 24 horas após a parição, estão em concordância com aqueles autores que demonstraram a existência de leucocitose por neutrofilia e eosinopenia imediatamente após a parição $1,5,7,8,13,14,16,22,23,25$. Discordou-se dos autores 4,11,12 que afirmaram que o puerpério não influenciava o leucograma.

O retorno dos valores do número total de leucócitos aos patamares vistos fora do puerpério ocorreu na primeira semana 
após a parição concordando com as observações de Hussain e Daniel ${ }^{7}$, Merril e Smith ${ }^{13}$ e Rakesh et al. ${ }^{15}$ que descreveram que esse retorno ocorreu entre o $5^{\circ}$ e $10^{\circ}$ dia de puerpério. Pelos resultados obtidos, discordou-se daqueles autores que constataram que esse retorno ocorria nas primeiras 24 horas ${ }^{6,8,14}$ ou entre o $15^{\circ}$ e $30^{\circ}$ dia após o parto ${ }^{13,26}$. Verificou-se, ainda, que durante a primeira semana do puerpério, a neutrofilia com desvio à esquerda regenerativo desaparecia, sendo que esta observação veio reforçar as constatações de Merril e Smith ${ }^{13}$ que descreveram, anteriormente, fato semelhante.

Nos primeiros dias do puerpério foi verificada uma linfocitose absoluta, sendo esse fenômeno descrito em duas pesquisas ${ }^{5,16}$. Essa constatação se contrapôs à relatada linfopenia relativa que ocorria no parto $^{13,26}$ e que se acentuava nos primeiros dias de puerpério ${ }^{13}$ ou mesmo naquelas que afirmaram que o número de linfócitos não sofria influência do puerpério ${ }^{9,14}$.

O quadro leucocitário, durante todo o período estudado, foi predominantemente linfocitário, sendo que esse fato, também, pode ser verificado na pesquisa brasileira realizada por Fagliari et al. ${ }^{5}$. Nas pesquisas, nas quais as amostras de sangue eram provenientes de animais criados no hemisfério norte, durante a parição ou puerpério, observou-se que esse predomínio não existia ${ }^{9,13}$ ou era predominantemente neutrofílico ${ }^{8,22}$. Justificou-se tais diferenças como decorrentes das particularidades do quadro hemático de bovinos criados em regiões tropicais e subtropicais, como o Brasil, regiões nas quais os bovinos são naturalmente premunidos contra $\mathrm{O}$ Anaplasma sp e Babesia sp. Afora as possíveis inter-relações entre a linfocitose absoluta, encontrada nos primeiros dias do puerpério, com aquela referida em animais infectados cronicamente pelo Anaplasma sp e Babesia sp, não se encontrou qualquer outra razão que justificasse essa particularidade do leucograma de bovinos recém-paridos criados no Brasil, sendo necessário a realização de outras pesquisas que procurem elucidar a questão.

Finalizando as modificações observadas no leucograma durante o período pós-parto, encontrou-se na literatura compulsada a existência de diversos autores que demonstraram a ocorrência de eosinopenia durante a parição ${ }^{1,8,13,22,23,26}$, fato, também, verificado na presente pesquisa.

Apesar do número de eosinófilos sofrer oscilação nos primeiros dias do puerpério, foi possível demonstrar que os valores obtidos nos primeiros dois dias são significativamente menores, do que os observados em vacas cujas amostras de sangue foram colhidas com mais de 60 dias após o parto. Demonstrou-se, também, que após as oscilações observadas nos primeiros dias após o parto, ocorria um aumento gradual do seu número até 90 dias após o parto. Dessa forma, concordou-se com os autores que verificaram que durante a evolução do puerpério deixa de ser observado a eosinopenia ${ }^{6,8}$, havendo divergência se isso ocorria no puerpério recente (até dois dias após o parto), ao final do puerpério clínico (por volta de 20 dias) ou se somente após o final do puerpério (com mais de 28 dias após o parto). Por outro lado, discordou-se de pesquisas que descreveram que o número de eosinófilos não sofria influência do período pós-parto ${ }^{16}$.

A partir do final da primeira semana do puerpério o leucograma não sofreu maiores variações, exceção feita ao número de eosinófilos. Assim, discordou-se dos autores que afirmaram que, entre o $10^{\circ} \mathrm{e}$ $32^{\circ}$ dia após o parto, ocorria aumento do número de neutrófilos ${ }^{7}$ ou que, entre o $5^{\circ} \mathrm{e}$ $15^{\circ}$ dia após o parto, existia aumento do número de linfócitos ${ }^{6}$.

Em relação ao número absoluto de monócitos e basófilos, não foi possível determinar a natureza da influência para explicar a oscilação dos valores que oscilaram sem qualquer tendência de aumento ou diminuição dos valores. Na literatura compulsada há controvérsia sobre a existência dessa influência no número de 
monócitos, pois alguns autores ${ }^{5,13,16}$ não encontraram alterações significativas nos valores do número absoluto de monócitos, enquanto outros ${ }^{6,8}$ observaram que nos dois primeiros dias do puerpério os valores eram maiores do que os observados na fase final do puerpério. Com relação ao número absoluto de basófilos não foram encontradas maiores referências sobre possíveis variações relacionadas ao puerpério e restringiram-se somente a descrição dos resultados obtidos ${ }^{5,8,9}$.

Ao comparar os valores do leucograma obtidos na primeira semana do puerpério foi verificado que o número de leucócitos, neutrófilos bastonete, neutrófilos segmentado, total de neutrófilos e linfócitos foram significativamente maiores do que os valores de referência apresentados para bovinos adultos ( $>24$ meses) das raças Jersey, Nelore, Holandesa, Girolando e Gir, criados no Estado de São Paulo ${ }^{1,2,3}$. Diante da magnitude das modificações do leucograma observadas na primeira semana após o parto, recomenda-se para este período a adoção de valores de referência específicos, conforme apresentados no capítulo de resultados.

\section{Conclusões}

A análise, interpretação e discussão dos resultados obtidos na presente pesquisa, na qual procurou-se avaliar a influência do período pósparto sobre o leucograma de fêmeas bovinas da raça Holandesa, possibilitaram as conclusões a seguir detalhadas:

- durante todo o período estudado o quadro foi predominantemente linfocitário;

- verificou-se, em amostras colhidas com menos de 24 horas após o parto, que o quadro leucocitário foi caracterizado por leucocitose por neutrofilia com desvio à esquerda regenerativo associado a linfocitose e eosinopenia absoluta;

- na primeira semana após o parto, ocorria diminuição gradual do número de leucócitos, devido à diminuição do número absoluto de neutrófilos e linfócitos, sendo que, exceção feita ao número absoluto de eosinófilos, nas amostras colhidas entre 6 - 8 dias após o parto, os valores dessas variáveis haviam retornado aos patamares observados nos animais fora do puerpério;

- diante da magnitude das modificações do quadro leucocitário na primeira semana após o parto, recomenda-se a adoção de valores de referência específicos.

\section{The influence of postpartum period on the leucogram of Holstein cows}

\section{Abstract}

With the purpose to evaluate the influence of postpartum period on the values of the leucogram of Holstein cows, 142 blood samples were collected from cows during the first 90 days after parturition. Blood samples were collected from clinically healthy cows that were non-reactive to the antigen of bovine leukosis virus (GP 51). These animals were divided into 9 experimental groups based on the time of the postpartum sample collection. In the blood samples the following tests were done: counting of the total and differential number of leukocytes. During the entire study period, the leukocyte profile was predominantly lymphocytic, having a physiologic leukocytosis due to neutrophilia associated with a left regenerative shift, lymphocytosis, and eosinophilia during the first $24 \mathrm{~h}$ after parturition. During the first week after parturition, there was a gradual decrease in the number of leukocytes due to an absolute reduction in the total number of neutrophils and lymphocytes. An exception was observed with the absolute number of eosinophils from the samples collected between 6 - 8 days postpartum, the values of these variables were similar to those animals that had already completed puerperium.
Key-words: Leucogram. Hematology. Postpartum. Bovine. Holstein. 
During the first week of puerperium the values reference range between $18,977 \pm 5,644$ and $12,247 \pm 5,058$ leukocytes $/ \mathrm{mm}^{3} ; 8,453 \pm 2,956$ and 4,376 \pm 4,139 neutrophils $/ \mathrm{mm}^{3}$ (532 \pm 547 and $88 \pm 174$ band neutrophils $/ \mathrm{mm}^{3} ; 7,920 \pm 2,689$ and 4,289 \pm 4,039 segmented neutrophils $\left./ \mathrm{mm}^{3}\right) ; 334 \pm 387$ and $225 \pm 298$ eosinophils $/ \mathrm{mm}^{3} ; 50$ \pm 128 and $23 \pm 45$ basophils $/ \mathrm{mm}^{3} ; 10,004 \pm$ 4,901 and 7,595 \pm 3,127 lymphocytes $/ \mathrm{mm}^{3} ; 121 \pm 178$ and $21 \pm 85$ monocytes $/ \mathrm{mm}^{3}$.

\section{Referências}

1 BIRGEL JUNIOR, E. H. Hemograma de bovinos (Bos taurus, Lennaeus, 1758) da raça Jersey, criados no Estado de São Paulo. Influência de fatores etários, sexuais e da Infecção por vírus da leucose bovina. 1991. $172 \mathrm{f}$. Dissertação (Mestrado em Patologia Clínica) Faculdade de Medicina Veterinária e Zootecnia, Universidade de São Paulo, São Paulo, 1991.

2 TÁVORA, J. P. F. Hemograma de bovinos das raças Gir, Girolando e Holandesa criados no Estado de São Paulo. Estabelecimento dos valores de referência e avaliação da influência de fatores de variabilidade raciais, etários e sexuais. 1998. 163 f. Tese (Doutorado em Clínica Veterinária) - Faculdade de Medicina Veterinária e Zootecnia, Universidade de São Paulo, São Paulo, 1998.

3 COSTA, J. N. Leucograma de Zebuínos (Bos indicus, Linnaeus, 1758) sadios da raça Nelore, criados no Estado de São Paulo. Influência de fatores etários e sexuais. 1994. 124 f. Dissertação (Mestrado em Clínica Veterinária) - Faculdade de Medicina Veterinária e Zootecnia, Universidade de São Paulo.

4 D'ANGELINO, J. L.; et al. Influência da gestação e do puerpério sobre o quadro hemático de bovinos da raça Holandesa Branca e Preta. Revista da Faculdade de Medicina Veterinária e Zootecnia da Universidade de São Paulo, v. 14, p. 11-21, 1977.

5 FAGLIARI, J. J.; et al. Constituintes sangüíneos de vacas das raças Nelore (Bos indicus) e Holandesa (Bos taurus) e de bubalinos (Bubalus bubalis) da raça Murrah durante a gestação, no dia do parto e no puerpério. Arquivo Brasileiro de Medicina Veterinária e Zootecnia, v. 50, n. 3, p. 273-282, 1998.

6 GUIDRY, A. J.; PAAPE, M. J.; PEARSON, R. E. Effects of parturition and lactation on blood and milk cell concentrations, corticosteroids, and neutrophil phagocytosis in the cow. American Journal Veterinary Research, v. 37, n. 10, p. 1195-1200, 1976.

7 HUSSAIN, A. M.; DANIEL, R. C. W. Phagocytosis by uterine fluid and blood neutrophils and hematological changes in postpartum cows following normal and abnormal parturition. Theriogenology, v. 37, p. 1253$1267,1992$.

8 STRAUB, O. C.; et al. Bovine hematology. II. Effect of parturition and retention of fetal membranes on blood morphology. Journal American Veterinary Medicine
Association, v. 15, p. 618-622, 1959.

9 FERGUSON, L. C.; IRWIN, M. R.; BEACH, B. A. The effect of parturition on the blood picture of cows in health and during infection with Brucella abortus. American Journal Veterinary Research, v. 2, p. 394339, 1941.

10 GILBERT, R. P.; et al. Effect of parity on periparturient neutrophil function in dairy cows. Veterinary Immunology and Immunopathology, v. 36, p. 75-82, 1993.

11 KHAJURIA, R. R.; RAZDAN, M. N. Leucocytic picture under different physiological states. The Indian Veterinary Journal, n. 43, p. 886-892, 1966.

12 MEHERE, Y. S.; et al. Haematological and trace element profile during peripartum period in crossbred cows. Indian Journal of Animal Sciences, v. 72, n. 2, p. 148-150, 2002.

13 MERRIL, W. G.; SMITH, V. R. A comparison of some cellular and chemical constituents of blood at time of parturition and after administration of adrenocorticotrophin. Journal Dairy Science, v. 37, n. 5, p. 546-551, 1954.

14 MORRIS, P. G. D. Blood picture of a cow during a normal pregnancy and parturition. The Veterinary Journal, v. 100, p. 225-233, 1944.

15 RAKESH K.; et al. Status of haemogram, plasma proteins, minerals and sub-clinical ketosis in cows and buffaloes. Indian Journal of Animal Sciences, v. 71, n. 2, p. 118-121, 2001.

16 SILVA, F. M.; et al. Assessment of blood neutrophil oxidative burst activity in dairy cows during the period of parturition. Animal Science, n. 67, p. 421-426, 1998.

17 BIRGEL, E. H. Hematologia clínica veterinária In: BIRGEL, E. H.; BENESI, F. J. Patologia clínica veterinária. $2^{\circ}$ ed. São Paulo: Sociedade Paulista de Medicina Veterinária, 1982. $260 \mathrm{p}$.

18 BIRGEL, E. H. Leucose enzoótica dos bovinos adultos: aspectos clínicos e diagnósticos In: BIRGEL, E. H.; BENESI, F. J. Patologia clínica veterinária. $2^{\circ}$ ed. São Paulo: Sociedade Paulista de Medicina Veterinária, 1982. p. 249-260.

19 D'ANGELINO, J. L. Leucose enzoótica dos bovinos. Estudo retrospectivo da performance produtiva e reprodutiva de animais infectados e não infectados. 1991. p. 85. Tese (Livre-docência) - Faculdade de 
Medicina Veterinária e Zootecnia, Universidade de São Paulo, São Paulo,1991.

20 SAS. SAS. Cary, 2000. Software v. 8.1.

21 BERQUÓ, E. S.; SOUZA, J. M. P.; GOTLIEB, S. L. D. Bioestatística. São Paulo: Ed. Pedagógica e Universitária, 1980. 325 p.

22 BIRGEL JUNIOR, E. H.; GRUNERT, E. Avaliação da modificações da crase sangüínea nas últimas 96 horas de gestação em bovinos da raça Holandesa Preta e Branca. In: CONGRESSO BRASILEIRO DE MEDICINA VETERINARIA, 24., 1996, Goiânia, GO. Anais... Goiânia: Sociedade Goiana de Veterinária, 1996. p. 32-33.

23 PATERSON, J. Y. F. 17-hydroxycorticosteroids and leucocytes in the blood of dairy cattle. Journal Comparative Pathology, v. 67, p. 165-179, 1957.

24 PAAPE, M. J.; SCHULTZE, W. D.; MILLER, R. H. Leukocytic response to adrenocorticotrophic hormone as influenced by the infectious history of the mammary gland. Journal of Dairy Science, v. 56, n. 6, p. 733737, 1973.

25 HOLMANN, H. H. The blood picture of the cow. British Veterinary Journal, v. 111, p. 440-457, 1955.

26 GREATOREX, J. C. Observations on the haematology of calves and various breeds of adult dairy cattle. The British Veterinary Journal, v. 113, p. 65-70, 1957. 